\title{
Probing the non-thermal emission in the Perseus cluster with the JVLA
}

\author{
M. Gendron-Marsolais ${ }^{1}$, J. Hlavacek-Larrondo ${ }^{1}$, R. J. van Weeren ${ }^{2}$, \\ T. Clarke ${ }^{3}$, A. C. Fabian ${ }^{4}$ H. T. Intema ${ }^{2}$, G. B. Taylor ${ }^{5}$, \\ K. M. Blundell ${ }^{6}$ and J. S. Sanders ${ }^{7}$ \\ ${ }^{1}$ Département de Physique, Université de Montréal, Montréal, QC H3C 3J7, Canada \\ email: marie-lou@astro.umontreal.ca \\ ${ }^{2}$ Leiden Observatory, Leiden University, Niels Bohrweg 2, NL-2333CA, Leiden, \\ The Netherlands \\ ${ }^{3}$ Naval Research Laboratory, Code 7213, 4555 Overlook Ave. SW, Washington, DC 20375, USA \\ ${ }^{4}$ Institute of Astronomy, University of Cambridge, Madingley Road, Cambridge CB3 0HA \\ ${ }^{5}$ Department of Physics and Astronomy, University of New Mexico, Albuquerque, \\ NM 87131, USA \\ ${ }^{6}$ University of Oxford, Astrophysics, Keble Road, Oxford OX1 3RH, UK \\ ${ }^{7}$ Max-Planck-Institut für extraterrestrische Physik, 85748 Garching, Germany
}

\begin{abstract}
We present deep low radio frequency (230-470 MHz) observations from the Karl G. Jansky Very Large Array of the Perseus cluster, probing the non-thermal emission from the old particle population of the AGN outflows. Our observations of this nearby relaxed cool core cluster have revealed a multitude of new structures associated with the mini-halo, extending to hundreds of kpc in size. Its irregular morphology seems to have been influenced both by the AGN activity and by the sloshing motion of the cluster' gas. In addition, it has a filamentary structure similar to that seen in radio relics found in merging clusters. These results illustrate the high-quality images that can be obtained with the new JVLA at low radio-frequencies.
\end{abstract}

Keywords. galaxies: clusters: Perseus cluster, cooling flows, radio continuum: galaxies, X-rays: galaxies: clusters

\section{Introduction}

Jets created from accretion onto supermassive black holes (SMBHs) release relativistic particles on large distances. These strongly affect the intracluster medium (ICM) when located in the center of a brightest cluster galaxy (BCG). These jets inflate bubbles, displacing the ICM and creating regions of depleted X-ray emission. The bubbles, filled with relativistic plasma (i.e., radio lobes), are often discernible at $\sim \mathrm{GHz}$ frequencies. On the other hand, the hierarchical merging of subclusters and groups, from which cluster originate, also generates perturbations into the ICM through shocks and turbulence, constituting a potential source of reacceleration for these particles.

In the Perseus cluster, we observe several types of disturbances in the ICM that have been probed in detail by extensive X-ray observations. At least two pairs of X-ray cavities have been identified: the first pair, located at $5<r<20 \mathrm{kpc}$ from the AGN, is filled with radio emission (Böhringer et al. 1993) and the second, located further out at $25<$ $r<45 \mathrm{kpc}$ from the AGN, is devoid of radio emission above $\sim 1 \mathrm{GHz}$ (BranduardiRaymont et al. 1981; Fabian et al. 1981; Churazov et al. 2000). Inflated by generations of outbursts from the central AGN, these constitute the imprint of radio mode feedback, 
injecting energy into the ICM and compensating its radiative losses (e.g. Bîrzan et al. 2004; Dunn \& Fabian 2006; Rafferty et al. 2006). Beyond cavities are quasi-spherical ripples interpreted as sound waves (Fabian et al. 2003), as well as a semicircular cold front, two new elliptical cavities interpreted as potential ghost bubbles, two large regions of weak X-ray luminosity (the northern trough and the southern bay) and a loop-like structure above a long $\mathrm{H} \alpha$ filament (Fabian et al. 2011).

The Perseus cluster harbours a mini-halo (Soboleva et al. 1983; Pedlar et al. 1990; Burns et al. 1992; Sijbring 1993), a faint diffuse source of radio emission detected so far in about thirty cool core clusters (Giacintucci et al. 2017, see Feretti et al. 2012 for a review). The X-rays and mini-halo structure of Perseus have been compared in Fabian et al. (2011). This emission differs from that filling the X-ray cavities, being $\gtrsim 3$ times radio fainter and having a steeper spectral index $\left(\alpha<-1\right.$ for $S(\nu) \propto \nu^{\alpha}$, where $S$ is the flux density and $\nu$ is the frequency, Giacintucci et al. 2014a). Since the radiative timescale of the electrons is much shorter than the time required for them to reach the extent of these sources, the origin of mini-halos remains unclear. Two possible mechanisms have been proposed in the literature to explain the mini-halo emission: it might originate from the reacceleration of pre-existing electrons by turbulence (Gitti et al. 2002, 2004) or from the generation of new particles from inelastic collisions between relativistic cosmic-ray protons and thermal protons (e.g. Pfrommer \& Enßlin 2004). Simulations seem to suggest that turbulence created by sloshing motions of the cold gas in the core region is sufficient to re-accelerate electrons (e.g. ZuHone et al. 2013). A key prediction of these simulations is that mini-halos should be bounded by cold fronts.

Here we summarize results presented in Gendron-Marsolais et al. (2017) on new, deep Karl G. Jansky Very Large Array (JVLA) observations of the Perseus cluster in the P-band (230-470 MHz). The resolution and sensitivity of these data provide a detailed and extended view of the mini-halo structure, on which we focus our analysis. The recent update of the facilities with the EVLA project offer new abilities to study this structure.

We assume a redshift of $z=0.0183$ for NGC 1275 corresponding to a luminosity distance of $78.4 \mathrm{Mpc}$, assuming $H_{0}=69.6 \mathrm{~km} \mathrm{~s}^{-1} \mathrm{Mpc}^{-1}, \Omega_{\mathrm{M}}=0.286$ and $\Omega_{\mathrm{vac}}=0.714$. This corresponds to an angular scale of $22.5 \mathrm{kpc} \operatorname{arcmin}^{-1}$.

\section{JVLA observations of the Perseus cluster}

We obtained a total of 5 hours in the P-band (230-470 MHz) of the Karl G. Jansky Very Large Array in B-configuration on November 24 $4^{\text {th }}, 2013$ (project 13B-026). With the new broadband low frequency receivers, the P-band bandwidth has been widened from 300-340 MHz to 230-470 MHz, increasing significantly the sensitivity of the telescope. The resolution of these observations probes the faint extended emission of the mini-halo structure in the Perseus cluster. The data reduction was performed with CASA (Common Astronomy Software Applications, version 4.6). A pipeline was specifically developed to account for the strong presence of radio frequency interference (RFI) at low-frequencies and the extremely bright central AGN in Perseus outshining faint structures.

\section{Results}

Figure 1 shows the central part of the B-configuration final map obtained from the data reduction and imaging process described in Section 2. The resulting image has a rms of $0.35 \mathrm{mJy} /$ beam, peak at $10.63 \mathrm{Jy} /$ beam and a beam size of $22.1^{\prime \prime} \times 11.3^{\prime \prime}$. Beyond the central emission from NGC 1275, the large field of view includes NGC 1265, a wideangle tail radio galaxies, and IC 310, a radio galaxy with a potential blazar-like behavior (Aleksić et al. 2014b; Ahnen et al. 2017), both discovered by Ryle \& Windram (1968).The 


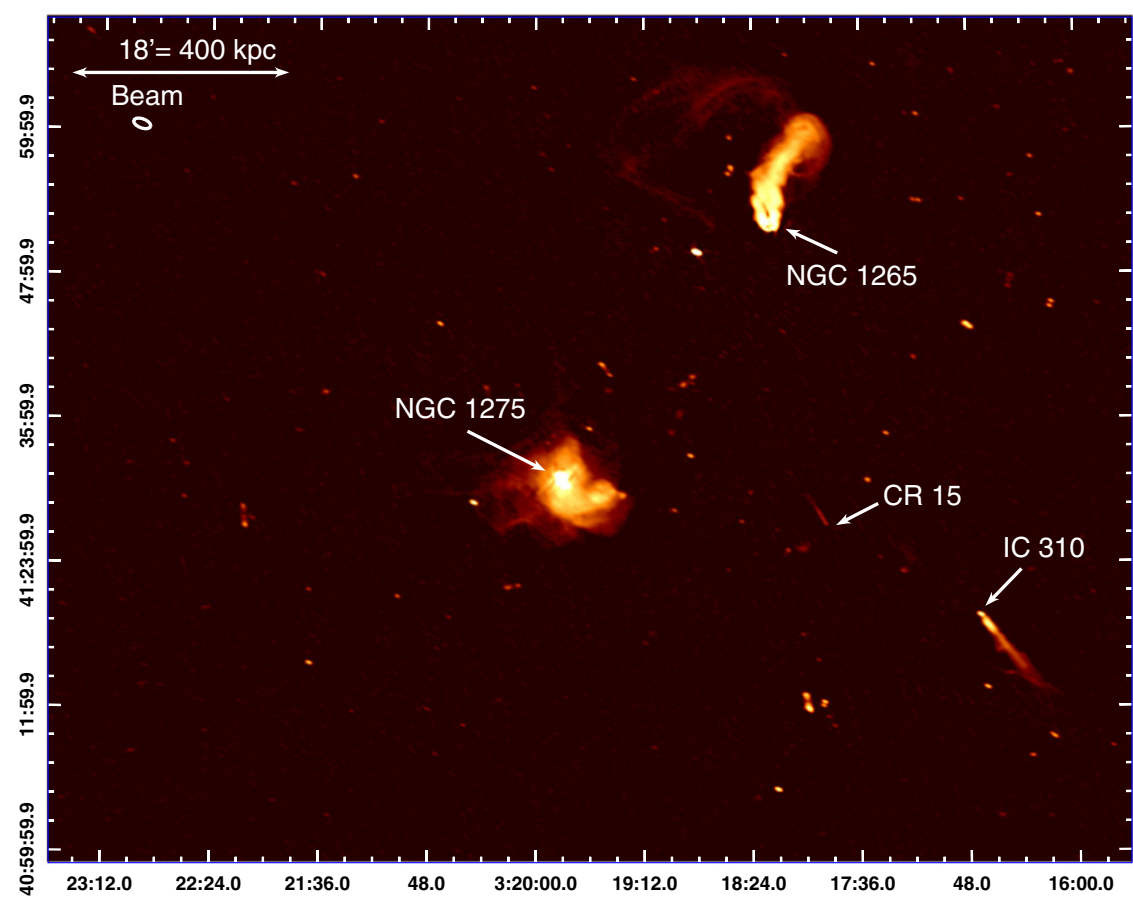

Figure 1. The central $2^{\circ} \times 1.5^{\circ}$ of the total field of view of the JVLA $230-470 \mathrm{MHz}$ radio map obtained in B-configuration. NGC 1275 is the bright source in the middle of the image. Tow wide-angle tail radio galaxies, NGC 1265 (NNW of NGC 1275) and CR 15 (between NGC 1275 and IC 310) as well as IC 310 (WSW of NGC 1275) are clearly visible. The resulting image has a rms noise of $0.35 \mathrm{mJy} /$ beam, a beam size of $22.1^{\prime \prime} \times 11.3^{\prime \prime}$ and a peak of $10.63 \mathrm{Jy} / \mathrm{beam}$.

analysis of their complex morphologies will be presented in future work (GendronMarsolais et al. 2017 in prep.). The smaller head-tail source CR 15 is also found between NGC 1275 and IC 310, the tail pointing in a northeast direction (Miley et al. 1972).

Figure 2 shows a zoom of the central radio emission surrounding NGC 1275. Features running roughly north-west/south-east through the core are the remaining artifacts due to some problematic antennas all located in the same arm of the JVLA. The diffuse minihalo structure extends up to $\sim 150 \mathrm{kpc}$ from the AGN and shows a complex structure. The general shape of the mini-halo in the Perseus cluster has an irregular morphology, curving counterclockwise. It is also elongated in the direction of the radio bubble system. In addition to this large-scale structure shape, several fine structure details in the emission have been identified: two spurs are seen to the east and southeast of the AGN, an extension to the north, an edge to the south-west, a plume of emission to the south-south-west and a concave edge to the south.

\section{Discussion}

\subsection{Comparison with previous radio observations}

Figure 3 shows the $74 \mathrm{MHz}, 235 \mathrm{MHz}, 230-470 \mathrm{MHz}$ and $610 \mathrm{MHz}$ radio contours overlaid on the fractional residual X-ray image. The mini-halo is seen clearly at 230-470 $\mathrm{MHz}$ and $610 \mathrm{MHz}$, while only spurs of radio emission extend outside the X-ray cavities at $74 \mathrm{MHz}$ and almost no emission is seen outside the inner cavities at $235 \mathrm{MHz}$ with the GMRT image at these sensitivity levels. Mini-halos generally have steep spectral indexes but the noise levels of the 74 and $235 \mathrm{MHz}$ images ( 80 and 10mJy/beam, respectively) are 


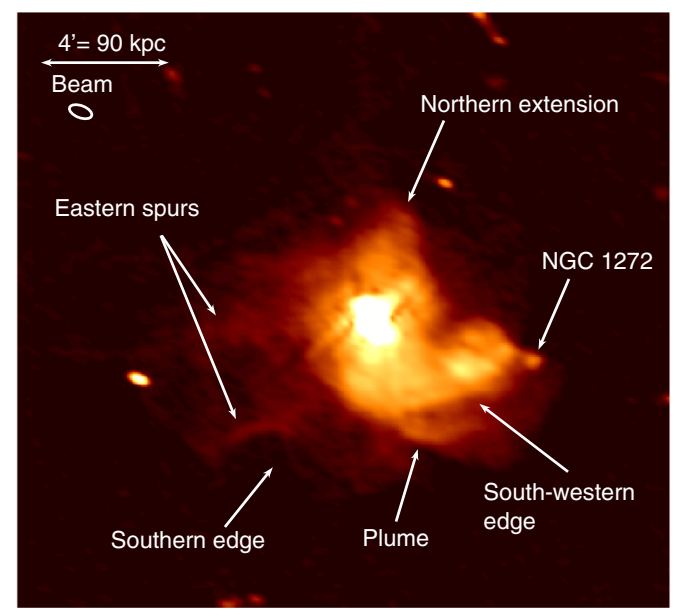

Figure 2. A zoom on the emission surrounding NGC 1275 from the $230-470 \mathrm{MHz}$ radio map seen in figure 1. The main structures of the mini-halo are identified: the northern extension, the two eastern spurs, the concave edge to the south, the south-western edge and a plume of emission to the south-south-west. The small knob at the end of the western tail is the galaxy NGC 1272.

too high for the faint mini-halo to be detected. The new JVLA facilities have produced an order of magnitude deeper image than the previous $330 \mathrm{MHz}$ VLA data $\left(\sigma_{\mathrm{rms}}=\right.$ $7 \mathrm{mJy} /$ beam) allowing the detection of the mini-halo emission to much larger radii and in much finer detail. The northern extension of emission as well as hints of the presence of the southern and south-western edges were already visible in $610 \mathrm{MHz}$ WSRT contours from Sijbring (1993). The synthesized beam size of these observations $\left(29^{\prime \prime} \times 44^{\prime \prime}\right)$ being about five times larger than the beam size of our $230-470 \mathrm{MHz}$ observations $\left(22.1^{\prime \prime} \times 11.3^{\prime \prime}\right)$, it only probed blurred emission from the eastern spurs, the plume and the southern edge, not fine filaments or sharp edges compared to the new JVLA observations.

\subsection{Large-scale structure of the mini-halo}

The mini-halo - sloshing cold front correspondences found in many clusters support the reacceleration hypothesis according to which cooled relativistic electrons injected by past AGN activity are reaccelerated by turbulence that may be produced by sloshing motion (Gitti et al. 2002, 2004; Mazzotta \& Giacintucci 2008; ZuHone et al. 2013; Giacintucci et al. 2014a). The B-configuration radio contours starting at $5 \sigma$ are overlaid on Chandra X-ray image (Fabian et al. 2011) on Figure 4- left. One of the most striking features in the deep X-ray images of the Perseus cluster is the spiral pattern of the X-ray emitting gas. A similar trend is also clearly seen in the temperature map (Fabian et al. 2006, see figure 4- right) and the western part of it was identified as a cold front (Fabian et al. 2011), a sharp contact discontinuity between gas regions with different temperatures and densities. The characteristic spiral pattern of cold fronts are created by the sloshing of gas in a gravitational potential perturbed by a minor merger (see Markevitch \& Vikhlinin 2007 for a review). High-resolution simulations of cluster mergers also show that cold fronts are produced by minor mergers and can persist over gigayear time scales (Ascasibar \& Markevitch 2006). In the case of the Perseus cluster, a chain of bright galaxies visible to the west of the BCG NGC 1275 have been identified as the possible source of disturbance (Churazov et al. 2003). Interestingly, the curving shape of the mini-halo seems to be well aligned with the sloshing pattern. It matches 

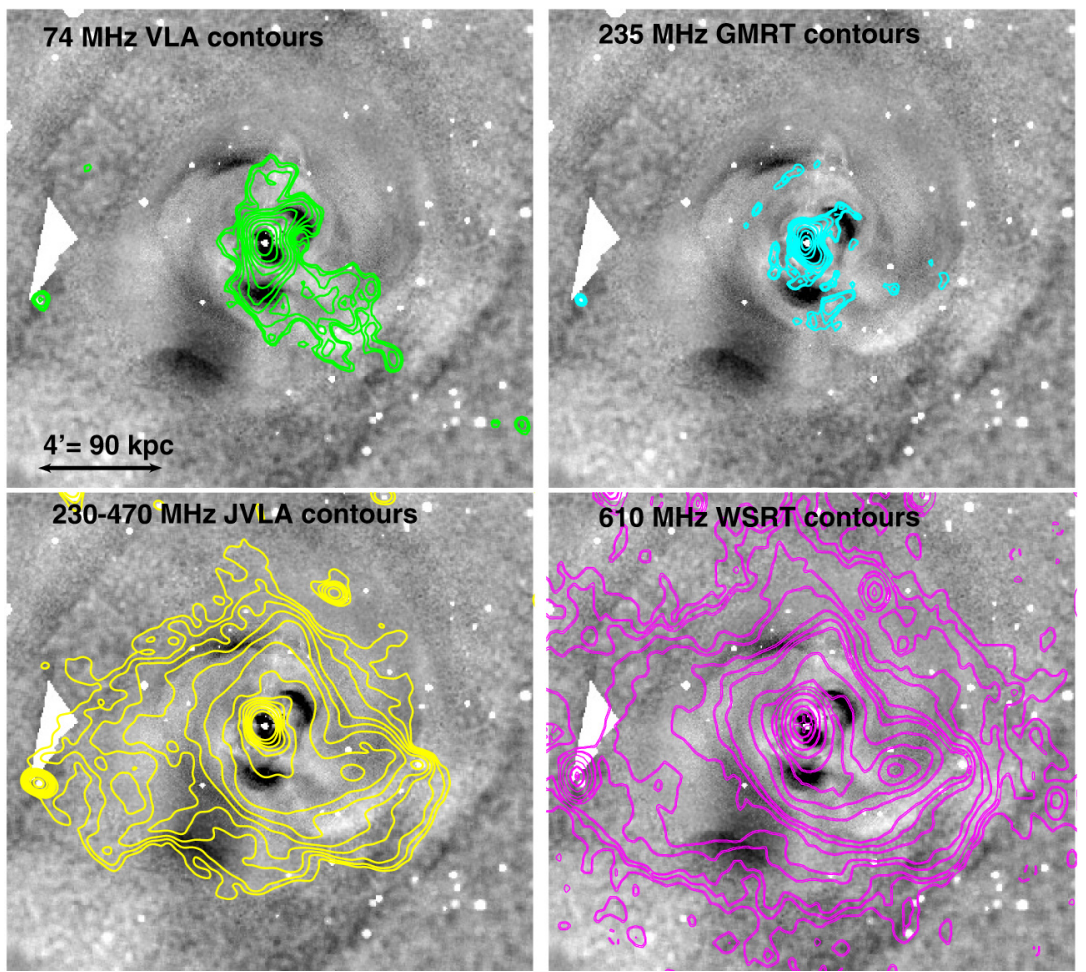

Figure 3. Chandra final composite fractional residual image from Fabian et al. (2011) in the $0.5-7 \mathrm{keV}$ band (total of $1.4 \mathrm{Ms}$ exposure) with radio contours at different frequencies overlaid. Top-left: $74 \mathrm{MHz}$ A configuration VLA contours (synthesized beamwidth of $24^{\prime \prime}, \sigma_{\mathrm{rms}}=80 \mathrm{mJy} /$ beam). A total of 11 contours are drawn, increasing logarithmically from 0.3 Jy/beam to $36.2 \mathrm{Jy} /$ beam (Blundell et al. 2002). Top-right: $235 \mathrm{MHz}$ GMRT contours (synthesized beamwidth of $13^{\prime \prime}$ ). 10 contours are drawn, increasing logarithmically from $5 \sigma_{\text {rms }}=50 \mathrm{mJy} /$ beam to $9 \mathrm{Jy} /$ beam. Bottom-left: $230-470 \mathrm{MHz}$ contours from the new JVLA B-configuration (beamwidth of $22.1^{\prime \prime} \times 11.3^{\prime \prime}$ ). A total of 13 contours are drawn, also increasing logarithmically from $3 \sigma=1.05 \mathrm{mJy} /$ beam to $1 \mathrm{Jy}$. Bottom-right: $610 \mathrm{MHz}$ WSRT contours from Sijbring (1993), synthesized beamwidth of $29^{\prime \prime} \times 44^{\prime \prime}$ and $\sigma_{\mathrm{rms}}=0.4 \mathrm{mJy} /$ beam). The contours levels are -0.8 (dashed), 0.8, 1.6, 2.4 , 5, 7.5, 15, 22.5, 30, 60, 90, 120, 150, 300, 850, 2500, 5000 and $10000 \mathrm{mJy} / \mathrm{beam}$.

both the size and the direction of curvature (counterclockwise). However, the mini-halo is also elongated in the direction of the cavity system. This spatial correlation is consistent with the scenario that AGN feedback could contribute to the injection of turbulence in the ICM and reaccelerate the relativistic particles responsible for the mini-halo emission (Cassano et al. 2008).

Figure 4- middle shows the X-ray GGM filtered image with the same radio contours. The position of the western cold front is indicated by the arrows. This image shows very clearly how the mini-halo emission is mostly contained behind the cold front: there is a sharp edge in the radio image associated with the mini-halo, but the particles appear as well to leak out as there is an even fainter ( 2 to 3 times fainter) part of the mini-halo that extends beyond the cold front in the south-western direction.

Another interesting large scale structure present in the mini-halo of the Perseus cluster is the southern edge identified in figure 2. Compared with the X-ray observations (see figure 4- left), the radio emission seems to avoid the southern bay, an intriguing feature located about $100 \mathrm{kpc}$ south of the nucleus, first reported in Fabian et al. (2006). 

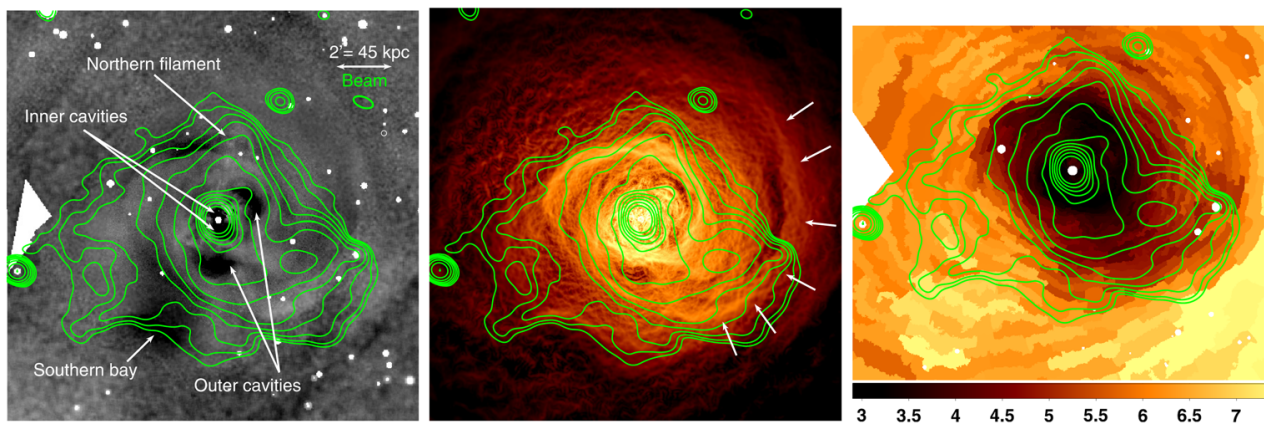

Figure 4. Left - Chandra final composite fractional residual image from Fabian et al. (2011) in the $0.5-7 \mathrm{keV}$ band (total of $1.4 \mathrm{Ms}$ exposure) with $230-470 \mathrm{MHz}$ contours from $5 \sigma=1.75$ mJy/beam to 1 Jy overlaid from JVLA B-configuration. Middle - GGM filtered image of the merged X-ray observations with Gaussian width $\sigma=4$ pixels (Sanders et al. 2016) with the same 230-470 MHz JVLA contours. The position of the western cold front is indicated with white arrows. Right - Central part of the temperature map of the Perseus cluster from Fabian et al. (2011) with signal-to-noise ratio of 150 with the same $230-470 \mathrm{MHz}$ JVLA contours. Units are $\mathrm{keV}$.

This 'bay' structure, founded in three nearby relaxed clusters (Perseus, Centaurus and Abell 1795, Walker et al. 2017), behaves like cold fronts but has the opposite curvature toward the interior of the cluster. According to simulations of gas sloshing, they might be resulting from Kelvin-Helmholtz instabilities (Walker et al. 2017).

\subsection{Filamentary structure}

Very few filamentary structures like the ones present in the P-band JVLA observations of the Perseus cluster have been observed before in mini-halos. In Abell 2626, two elongated, $\sim 5 \mathrm{kpc}$ thick, arc-like radio features with longitudinal extensions of $\sim 70 \mathrm{kpc}$ are detected in its mini-halo (Gitti et al. 2004; Gitti 2013). In the case of Perseus, the two eastern spurs identified in figure 2 are $\geq 10 \mathrm{kpc}$ thick and extend over $\sim 150 \mathrm{kpc}$ in scale. Interestingly, similar filaments are found in radio relics (e.g. the relic in Abell 2256, Owen et al. 2014), large elongated diffuse polarized radio sources located at cluster peripheries. Relics could result from synchrotron emission of electrons reaccelerated by mergers or accretion shocks (Ensslin et al. 1998; Brunetti \& Jones 2014; van Weeren et al. 2017). In Perseus though, no shocks corresponding with the position of the filaments are known. We can also speculate that these filaments trace regions of enhanced magnetic fields or locally enhanced turbulence. Alternatively, they could reflect the original distribution of fossil plasma, for example from an old AGN outburst (up to a Gyr ago) that are re-accelerated by turbulence or weak shocks.

As shown in figure 5, the northern extension of the mini-halo also matches the position of the northern filament seen in the $\mathrm{H} \alpha$ map from Conselice et al. (2001). The loop-like $\mathrm{X}$-ray structure extending at the end of the northern filament has been interpreted as fallback gas dragged out to the north by previously formed bubbles (Fabian et al. 2011). As for the elongated shape of the mini-halo aligned with the cavity system, the northern extension shows another correlation of the mini-halo with the relativistic jets. Therefore, the shape of mini-halos seems to originate both from sloshing and from past AGN activity.

\subsection{Implications for our understanding of mini-halos}

The deep JVLA observations of the Perseus cluster combined with the cluster's properties (proximity, brightness and sloshing plane inclination) have revealed lots of structures, 

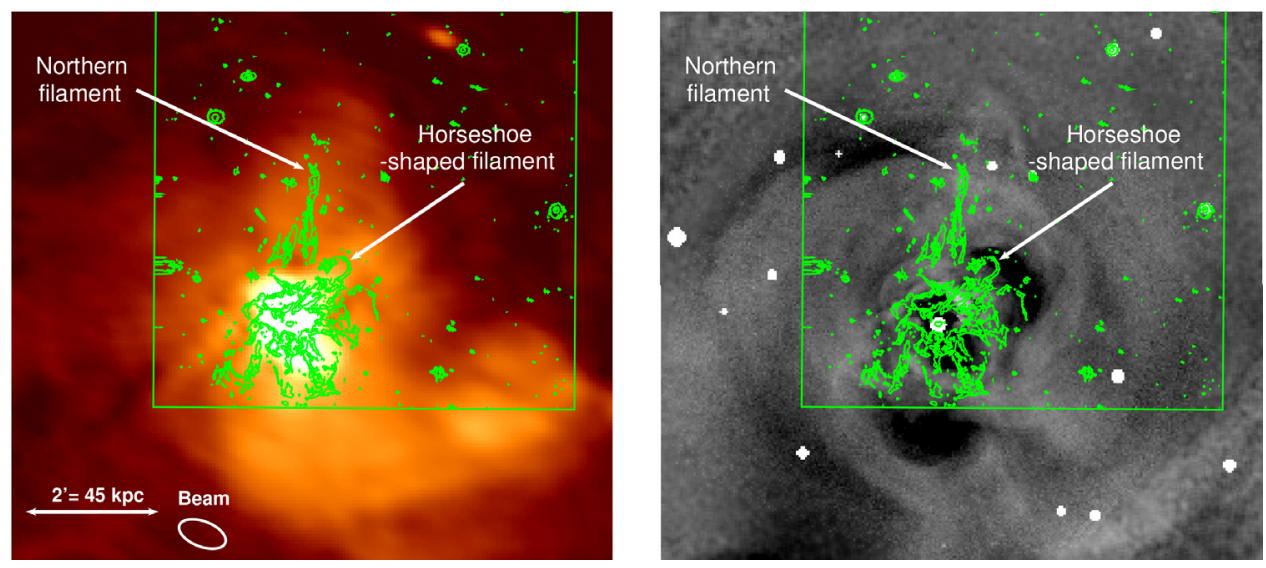

Figure 5. Left - JVLA 230-470 MHz B-configuration image with $\mathrm{H} \alpha$ contours (in green) of the continuum-subtracted $\mathrm{H} \alpha$ map from Conselice et al. (2001). The green region indicates the edge of the $\mathrm{H} \alpha$ image. The horseshoe-shaped filament and the northern filament are identified. Right - The fractional residual X-ray image with the same $\mathrm{H} \alpha$ contours.

unlike the present observations of most mini-halos which appear to be of fuzzy and uniform emission. This could be due to the resolution and sensitivity of the radio observations of mini-halos. Few mini-halos also present structures, e.g. the arc-like radio features in Abell 2626 (Gitti et al. 2004; Gitti 2013) or the spiral-shaped tail of emission in RX J1720.1+2638 with a length of $\sim 230 \mathrm{kpc}$ (Giacintucci et al. 2014b). Perseus's minihalo has similar size but a much higher flux density, 3 to 4 orders of magnitude higher than other mini-halos (Giacintucci et al. 2014a). Even with this level of detail, the emission is still mostly constrained behind the sloshing cold front, delimited by a sharp radio edge, providing a qualitative test of the reacceleration hypothesis. However, faint emission is also seen beyond this edge as if particles appear to leak out (see figure 4- middle). Again, as larger structures are resolved out in the B-array observations, we could still miss a large-scale diffuse mini-halo that would extend well beyond the cold front edge indicating that there must be a source of acceleration that goes beyond the cold front.

\section{Conclusions}

We present a detailed radio map of the Perseus cluster obtained from $5 \mathrm{~h}$ of observations with the JVLA at 230-470 MHz in the B-configuration. In summary, we conclude the following.

(a) Several structures have been identified in the mini-halo emission: the northern extension, two filamentary spurs to the east and a clear edge avoiding the X-ray southern bay. The general shape of the mini-halo is curving counterclockwise and is elongated in the direction of the cavity system. At 230-470 MHz, Perseus's mini-halo extends up to $135 \mathrm{kpc}$ from the nucleus and has a total flux density of $12.64 \mathrm{Jy}$.

(b) The comparison of the 230-470 MHz map with deep Chandra observations has shown that the mini-halo is enclosed mostly behind the western sloshing cold front, qualitatively supporting the reacceleration hypothesis. However, fainter emission is also seen beyond, as if particles leaking out.

(c) The large-scale and fine structure show a correlation of the mini-halo emission with both the sloshing motion and the relativistic jets of the AGN.

(d) Mysterious filamentary spurs of emission are found to the east, similar to radio relics, but no shocks corresponding with the position of the filaments are known. 
(e) The shape of the mini-halo resembles the central simulated synchrotron radiation in magnetohydrodynamic simulations of gas sloshing in galaxy clusters for example from ZuHone et al. (2013).

These results illustrate the high-quality images that can be obtained with JVLA at low radio-frequencies, as well as the necessity to obtain deeper, higher-fidelity radio images of extended emission in clusters to further understand their origin.

\section{References}

Ahnen, M. L. et al. 2017, A\&SA

Aleksić, J. et al. 2014b, A\&̊A, 563, A91

Ascasibar, Y., \& Markevitch, M. 2006, ApJ, 650, 102

Bîrzan, L., Rafferty, D. A., McNamara, B. R., Wise, M. W., \& Nulsen, P. E. J. 2004, ApJ, 607, 800

Blundell, K. M., Kassim, N. E., \& Perley, R. A. 2002, in Proc. IAU Coll. 199. p. 189

Böhringer, H., Voges, W., Fabian, A. C., Edge, A. C., \& Neumann, D. M. 1993, 264, L25

Branduardi-Raymont, G., Fabricant, D., Feigelson, E., Gorenstein, P., Grindlay, J., Soltan, A., \& Zamorani, G. 1981, 248, 55

Brunetti, G., \& Jones, T. W. 2014, 23, 1430007

Burns, J. O., Sulkanen, M. E., Gisler G. R., \& Perley, R. A. 1992, 388, L49

Cassano, R., Gitti, M., \& Brunetti, G. 2008, 486, L31

Churazov, E., Forman, W., Jones, C., \& Böhringer, H. 2000, A\&3A, 356, 788

Churazov, E., Forman, W., Jones, C., \& Böhringer, H. 2003, ApJ, 590, 225

Conselice, C. J., Gallagher, III J. S., \& Wyse, R. F. G. 2001, ApJ, 122, 2281

Dunn, R. J. H., \& Fabian, A. C. 2006, MNRAS, 373, 959

Ensslin, T. A., Biermann, P. L., Klein, U., \& Kohle, S. 1998, A\& A, 332, 395

Fabian, A. C., Hu, E. M., Cowie, L. L., \& Grindlay, J. 1981, ApJ, 248, 47

Fabian, A. C., Sanders, J. S., Allen, S. W., Crawford, C. S., Iwasawa, K., Johnstone, R. M., Schmidt, R. W., \& Taylor, G. B. 2003, MNRAS, 344, L43

Fabian, A. C., Sanders, J. S., Taylor, G. B., Allen, S. W., Crawford, C. S., Johnstone, R. M., \& Iwasawa, K. 2006, MNRAS, 366, 417

Fabian, A. C., et al. 2011, MNRAS, 418, 2154

Feretti, L., Giovannini, G., Govoni, F., \& Murgia, M. 2012, A\&ARv, 20, 54

Gendron-Marsolais, M., et al. 2017, MNRAS, 469, 3872

Giacintucci, S., Markevitch, M., Venturi, T., Clarke, T. E., \& Cassano, R. Pasquale Mazzotta 2014a, ApJ, 781, 9

Giacintucci, S., Markevitch, M., Brunetti, G., ZuHone, J. A., Venturi, T., Mazzotta, P., \& Bourdin, H. 2014b, ApJ, 795, 73

Giacintucci, S., Markevitch, M., Cassano, R., Venturi, T., Clarke, T. E., \& Brunetti, G. 2017, ApJ

Gitti, M. 2013, MNRAS, 436, L84

Gitti, M., Brunetti, G., \& Setti, G. 2002, A\&A, 386, 456

Gitti, M., Brunetti, G., Feretti, L., \& Setti, G. 2004, A\&A A, 417, 1

Markevitch, M., \& Vikhlinin, A. 2007, ApJ, 443, 1

Mazzotta, P., \& Giacintucci, S. 2008, ApJ, 675, L9

Miley, G. K., Perola, G. C., van der Kruit, P. C., \& van der Laan, H. 1972, Nature, 237, 269

Owen, F. N., Rudnick, L., Eilek, J., Rau, U., Bhatnagar, S., \& Kogan, L. 2014, ApJ, 794, 24

Pedlar, A., Ghataure, H. S., Davies, R. D., Harrison, B. A., Perley, R., Crane, P. C., \& Unger, S. W. 1990, MNRAS, 246, 477

Pfrommer, C., \& Enßlin, T. A. 2004, A\& A, 413, 17

Rafferty, D. A., McNamara, B. R., Nulsen, P. E. J., \& Wise, M. W. 2006, ApJ, 652, 216

Ryle, M., \& Windram, M. D. 1968, MNRAS, 138, 1

Sanders, J. S., Fabian, A. C., Russell, H. R., Walker, S. A., \& Blundell, K. M. 2016, MNRAS, 460, 1898

Sijbring, L. G. 1993, PhD thesis, Groningen Univ. 
Soboleva, N. S., Temirova, A. V., Timofeeva, G. M., \& Aliakberov, K. D. 1983, Soviet Astronomy Letters, 9, 305

Walker S. A., Hlavacek-Larrondo J., Gendron-Marsolais M., Fabian A. C., Intema H., Sanders, J. S., Bamford, J. T., \& van Weeren, R. 2017, MNRAS, 468, 2506

ZuHone, J., Markevitch, M., Brunetti, G., \& Giacintucci, S. 2013, ApJ, 762, 78

van Weeren, R. J. et al. 2017, Nature Astronomy, 1, 0005 